\title{
Correlation between Balance and BMI in Collegiate students: A cross sectional study
}

\section{Tejaswini Padmanabha Suvarna *1, Joseph Oliver Raj ${ }^{2}$, Nithin Prakash ${ }^{3}$.}

${ }^{* 1}$ Alva's College Of Physiotherapy, Moodbidri, Dk-574227, Karnataka, India.

${ }^{2}$ Dean, Abhinav Bindra Sports Medicine and Research Institute, Bhubaneswar, Odisha, India.

2 PhD Research Scholar, Vinayaka Mission's College of Physiotherapy, Vinayaka Mission's Research Foundation (Deemed To Be University), Salem - 636308, Tamilnadu, India.

${ }^{3}$ Assistant Professor, Alva's College Of Physiotherapy, Moodbidri, Dk-574227, Karnataka, India.

\section{ABSTRACT}

Background: Balance deficits are usually related to medial-lateral instability. BMI could be an important factor to consider as; excess body mass or increased accumulation of adipose tissue can directly impact the postural stability which in return impacts balance.

Purpose of the study: To find the correlation between BMI and Balance.

Method: Sample consisted of 149 students, out of which 100 (67\%) were males and 49 (33\%) were females. BMI was calculated and was categorized into groups. Bilateral limb length was measured for normalising the data. Static balance was measured by performing blinded stork test and dynamic balance by performing Ybalance test. Data was analysed using Pearson's correlation test.

Result: There was significant correlation between BMI and static balance of left leg $(r=0.713,95 \% \mathrm{Cl} 0.623$, $0.784, p=0.01$ ) but on comparison, there was no significant correlation between BMI and static balance of right leg $(r=0.0458,95 \% \mathrm{Cl}-0.11,-0.205, p=0.58)$. It was found that there was no significant correlation between $\mathrm{BMI}$ and Left Anterior $(r=-0.134,95 \% \mathrm{Cl}-0.289,-0.0274, \mathrm{p}=0.103)$, Left Posterolateral $(r=-0.0775,95 \% \mathrm{Cl}-$ $0.235,0.0843, p=0.347)$, Left Posteromedial ( $r=-0.0903,95 \% \mathrm{Cl}-0.248,-0.0715, p=0.273)$ respectively. Also, it was found that there was no significant correlation between BMI and Right Anterior $(r=-0.236,95 \% \mathrm{Cl}-0.382$, $-0.0778, p=0.00381)$, Right Posterolateral $(r=-0.193,95 \% \mathrm{Cl}-0.343,-0.0334, p=0.0183)$, Right Posteromedial $(r=-0.126,95 \% \mathrm{Cl}-0.281,-0.0354, p=0.125)$ respectively.

Conclusion: There was significant correlation between BMI and static balance of left leg and no correlation was established between BMI and static balance on right leg and also no correlation was established between BMI and static and dynamic balance for right and left leg.

KEY WORDS: Static balance, Dynamic balance, Body Mass Index, Obesity, Limb length.

Address for correspondence: Tejaswini Padmanabha Suvarna, Alva's College Of Physiotherapy, Moodbidri, Dk-574227, Karnataka, India. E-Mail: suvarnatejaswini011@gmail.com

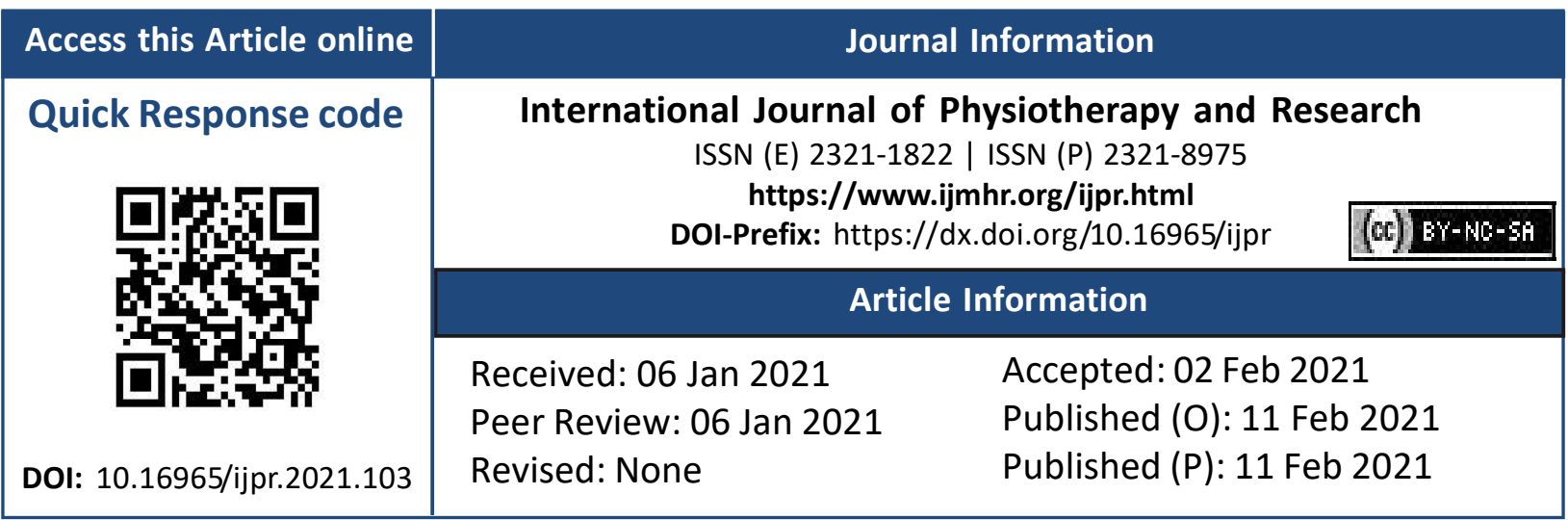

\section{INTRODUCTION}

Balance is characterized as the capacity to keep up the line of gravity of a body inside the base of support with negligible postural sway [1]. A specific development of influence is basic and unavoidable because of little perturbation inside the body (e.g., breathing, moving body weight for one foot to the next or from forefoot to hind foot) or from outside source (e.g., air flows, floor vibration, 
and so on). An increase in postural sway isn't really a marker of less balance to such an extent as it is a pointer of diminished neuromuscular control [2]. Keeping up balance requires coordination of contribution from various tactile frameworks including the vestibular, somatosensory and visual system [3].

Postural sway can happen in all planes of movement, which make it an undeniably troublesome capacity to restore. According to researches, there is a strong relation between decreased postural balance and stability, especially medial-lateral and potential risk of falling. To maintain balance, an individual standing should have the option to keep the vertical projection of their centre of mass inside their base of support, bringing about minimal average medial-lateral or anteriorposterior sway. Ankle sprains are one of the most common injuries among athletes and physically active individuals. The commonest residual disability after an ankle sprain is unsteadiness alongside sway of the body. Mechanical instability includes two factors, one being lack of stabilization structures and mobility beyond physiological limits. Functional unsteadiness or instability includes repetitive injuries to the ankle or a feeling of giving away [4]. Around $40 \%$ of the patients with ankle sprain portray excessive body sway and instability [5]. Injury to the ankle leads to lack of proprioception and hindered postural control. People with weakness of muscles and less postural control are more vulnerable to an injury of the ankle than those with better postural control.

Static balance is characterized by maintaining postural equilibrium whilst holding the body in a fixed position and dynamic balance is the maintenance of postural balance while other body parts are moving [6].

Functional balance assessments: Functional tests of balance emphasize on maintaining static and dynamic balance, regardless of whether it involves any perturbation or change of center of mass or during steady stance [7]. Standardized tests of balance are accessible to permit allied health care professionals to evaluate a person's postural control. Some of the functional balance tests which are accessible are Romberg Test [7],

Functional Reach Test [7], Berg Balance Scale [7], Performance-Oriented Mobility Assessment (POMA) [7], Timed Up and Go Test [7], Balance Efficacy Scale [7], Stork Test [8], Y- Balance Test [9], Star Excursion Test [10].

We performed blinded stork test for static balance \& Y-Balance test to asses dynamic balance.

Body Mass Index (BMI): The weight file (BMI), or Quetlet record, is a heuristic intermediary for human muscle to fat ratio dependent on a person's weight and height. Body Mass Index is characterized as the person's weight partitioned by the square of their height [11].

The body fat not just impacts an individual psychologically, but also functionally. The Body Mass Index (BMI), determined as weight (in kilograms)/tallness (in meters, squared), is the ordinarily utilized measurement to characterize the anthropometric height/weight attributes which represents the fatness of an individual, thus categorizing them into groups [12]. An expert group gathered in 1993 by World Health Organisation to build up the uniform categories of BMI and so was classified into four significant categories like underweight, normal, overweight and obese[12]. $\mathrm{BMI}$ is categorized into four groups: Underweight- BMI (<18.5), Normal- BMI (18.5 - 25), Overweight- BMI (25-30), Obese- BMI (>30).

A BMI of 18.5 to 25 may demonstrate ideal weight; a BMI lower than 18.5 proposes the individual is underweight while a number over 25 may show the individual is overweight; an individual may have a BMI below 18.5 because of sickness; a number above recommends the individual is large (more than 40, excessively obese). As discussed earlier, balance deficits are usually related to medial-lateral instability, BMI could be an important factor to consider, as excess body mass or increased accumulation of adipose tissue can directly impact the postural stability which in return impacts balance [13].

Studies have shown a fair correlation between balance and BMI. Aim is to find if there's any significant correlation between these two factors. 


\section{METHODOLOGY}

Sample consisted of 149 students, out of which 100 (67\%) were males and 49 (33\%) were females. The subject included normal healthy, co-operative individuals between the age group of 17 to 25 years. Individuals with recent fracture of trunk \& extremities, deformities of the lower limb, any neurological impairments, soft-tissue injuries preventing normal ROM and individuals with known balance issues were excluded from the study.

After getting ethical clearance from Institutional Ethical Committee, the samples were recruited using convenient sampling. A detailed explanation of aim, objective of the study \& methodology was given to the subjects. All the queries of the participants were answered \& consent was taken prior to the measurement of the required data. The weight of the subjects was taken from the weighing machine $\&$ height was taken from wall mounted stadiometer. BMI was calculated using the standard BMI calculation formula $\mathrm{BMI}=$ weight $(\mathrm{kg}) /$ height $\left(\mathrm{m}^{2}\right)$ and subjects were categorized into four groups as Underweight- BMI (<18.5), Normal- BMI (18.5 - 25), Overweight- BMI (25-30), Obese- BMI (>30) [13].

Static balance was assessed using Blinded Standing Stork Test for both the lower limbs. Subject was asked to remove the shoes and place the hands on the hips, then position the non-supporting foot against the inside knee of the supporting leg. The subject was then asked to close his/her eyes. The stopwatch was stopped if the hand(s) come off the hips, if the standing foot is moved or if the eyes were open. Test was carried out for three attempts. Time taken by the subject for each attempt was calculated in three attempts and average of all the three attempts was used for the analysis.

Dynamic Balance was assessed by Y-Balance test. Test was carried out on a non-slippery surface. Six feet long black tape was used to form a marking line in 3 directions - Anterior, Posteromedial, Posterolateral. The line was then overlapped by three measuring tapes in all three directions respectively. Subject was made to stand on one leg where the ball of the touching foot was placed in intersecting point of all the three lines. The subject has to reach as far as possible with the opposite leg in each direction for three times without losing balance and follow the same for all the directions. The first point of contact on the measuring tape was taken for the reading. The test was followed for both the lower limbs. Limb length was taken for both the limbs for the final calculation. Calculation involved taking mean of all the three reach distances. Data was then normalized by dividing it by limb length of the same leg \& then multiplying it by 100 .

\section{RESULTS}

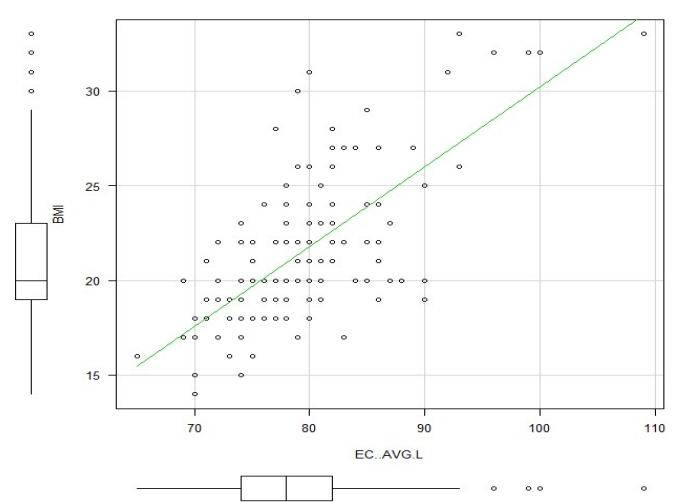

Figure 1 showing the correlation between $\mathrm{BMI}$ and Static Balance (Left)

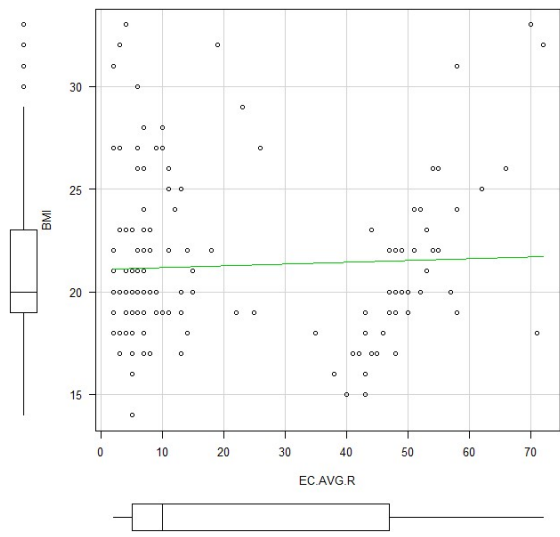

Figure 2 showing the correlation between BMI and Static Balance (Right)

Pearson's Correlation test was done to assess the correlation between balance and BMI. There was significant correlation between $\mathrm{BMI}$ and static balance of left leg $(r=0.713,95 \% \mathrm{Cl}$ $0.623-0.784, p=0.01$ ) but on comparison, there was no significant correlation between $\mathrm{BMI}$ and static balance of right leg $(r=0.0458,95 \%$ $\mathrm{Cl}-0.116-0.205, \mathrm{p}=0.58)$. Figure 1 and 2 represents the diagrammatic representation of the data. 
On analysing the relationship between BMI and Left Dynamic Balance, it was found that there was no significant correlation between BMI and Left Anterior ( $r=-0.134,95 \%$ $\mathrm{Cl}-0.289-0.0274, p=0.103)$, Left Posterolateral ( $r==-0.0775,95 \% \mathrm{Cl}-0.235-0.0843, p=0.347)$, Left Posteromedial $(r=-0.0903,95 \% \mathrm{Cl}-0.248$ $0.0715, p=0.273$ ) respectively. Figure 3,4 and 5 represents the diagrammatic representation of the data.

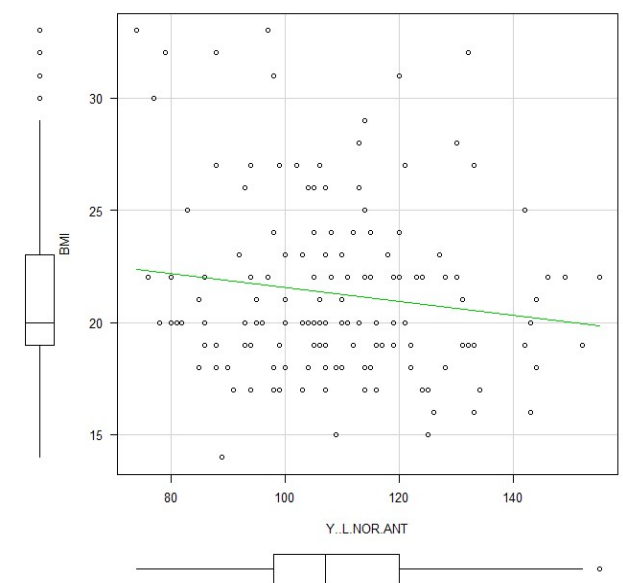

Figure 3 showing the correlation between BMI and Dynamic Balance (left- anterior)

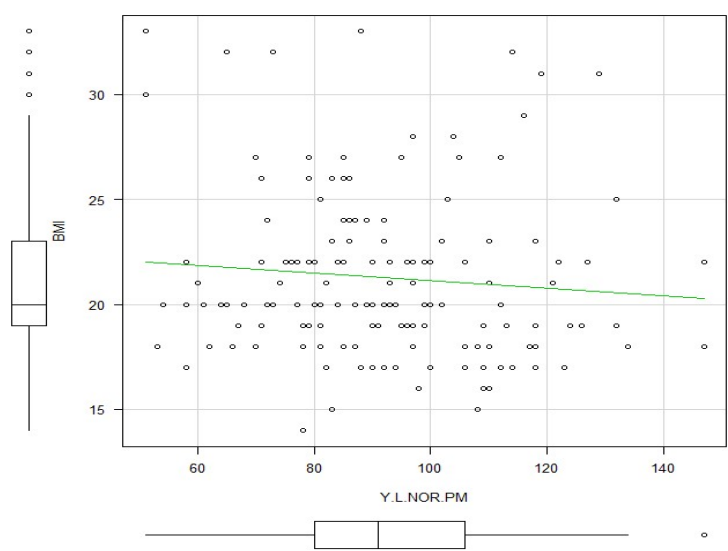

Figure 4 showing the correlation between $\mathrm{BMI}$ and Dynar

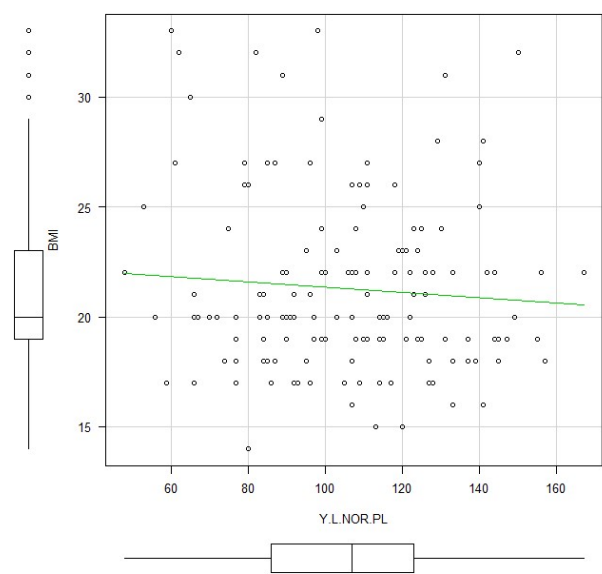

Figure 5 showing the correlation between BMI and Dynamic Balance (left- posterolateral)
On analysing the relationship between BMI and Right Dynamic Balance it was found that there was no significant correlation between BMI and Right Anterior ( $r=-0.236,95 \%$ $\mathrm{Cl}-0.382--0.0778, \mathrm{p}=0.00381$ ), Right Posterolateral $(r=-0.193,95 \% \mathrm{Cl}-0.343--0.0334$, $p=0.0183)$, Right Posteromedial $(r=-0.126$, $95 \% \mathrm{Cl}-0.281-0.0354, \mathrm{p}=0.125$ ) respectively. Figure 6, 7 and 8 represents the diagrammatic representation of the data.

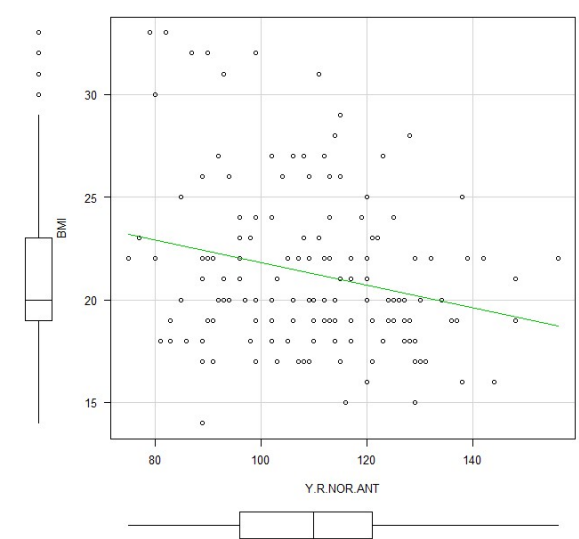

Figure 6 showing the correlation between BMI and Dynamic Balance (right- anterior)

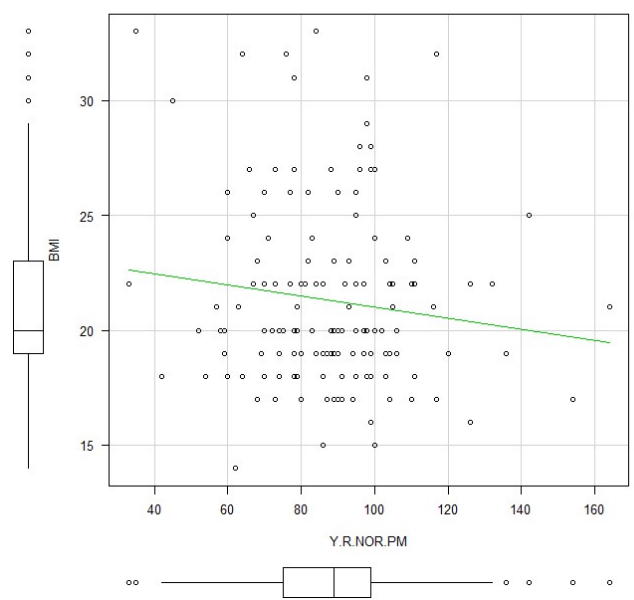

Figure 7 showing the correlation between $\mathrm{BMI}$ and Dynamic Balance (right- posteromedial)

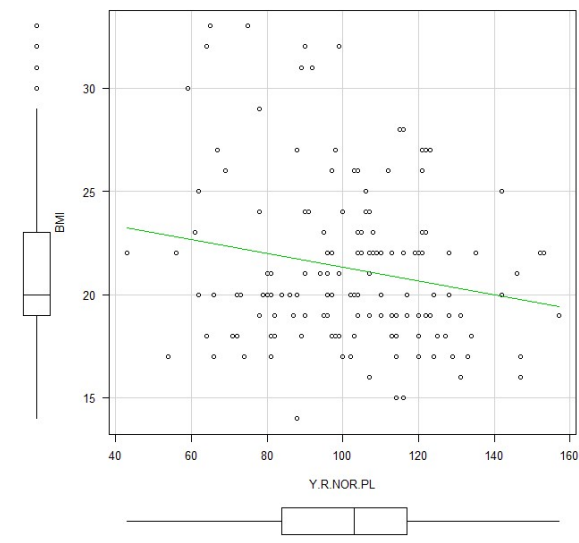

Figure 8 showing the correlation between BMI and Dynamic Balance (left- posterolateral) 


\section{DISCUSSION}

The study of balance \& BMI has revealed that amount of body fat affects static balance. The non-dominant limb has better balance compared to that of the dominant limb. The idea of limb dominance is based on the reason that the two hemispheres of the brain of the mind work distinctively and there is some particular utilization of one limb for activities under voluntary control. ${ }^{14}$ Lower limb dominance depends on the task to be performed. Depending on the need of the task when the subjects are asked to perform task(s) which involve whole body stabilization, there is switch towards the left leg [15]. So, as balance includes body stabilization, it very well may be expected that left leg i.e., non-predominant for roughly $96 \%$ of the subjects has a decent connection with the BMI when contrasted with that of the prevailing limb (right leg) demonstrating moderate or little correlation.

On comparison between BMI with dynamic balance, BMI has no correlation. As from the previous studies it was found that other factors such as visual feedback \& core stability play a major role when balancing in motion [16]. Dynamic balance causes the centre of gravity to move in response to muscular activity. This muscular activity may be influenced by an external or internal disturbance. During dynamic activity, the centre-of-pressure moves between the base of support boundaries and sometimes outside the base of support [16].

The core is important because it is defined as the lumbo-pelvic-hip complex where a person's centre of gravity is located and all movement begins [17]. Core muscles get activated in order to generate the rotational torque necessary and the motion of the extremities [18]. Also there is a need of proximal stability, that is the trunk and pelvis for the dynamic motion of the extremity [19]. The key is to control the body's centre of gravity, the point around which the body balances most perfectly. By keeping the centre of gravity between the bases of support, the subjects can more easily change direction. In addition, lowering the center of gravity adds stability. On the other hand, visual feedback stimulates proprioception in the subjects as they moved their own body weight and returned back to their original positions with stability, thereby increasing sensory nerve signal transmission $[20,21]$, followed by an increase in balance abilities and motor control of the muscles around the knee joints [22]. Also the equipment were not much suitable for replicating a dynamic environment to test the dynamic balance. Hence, the correlation may not have been accurate enough. Study could be performed with subjects with higher BMI, also on diseased population.

BMI has been a highly dominant factor in case of change in posture which then affects balance. Hence, an adequate amount of consideration needs to be given on maintaining the BMI within the normal range along with the other factors as to avoid the impairments related to balance.

\section{CONCLUSION}

BMI has an effect on the static balance, more significant on the non-dominant limb compared to the dominant limb as unilateral balancing requires whole body stabilization which causes an automatic shift to the non-dominant leg.

In case of dynamic balance, BMI has no significant effect. Rather, there are other factors such as Visual feedback and core stability that affect dynamic balance more than BMI. Dynamic stability of the body is maintained by keeping the centre of gravity between the base of support which involves proximal stability which is carried out by the core muscles. Lack of visual feedback and motor control of the muscles around knee joint can definitely disturb the balance of the body when in motion.

\section{Conflicts of interest: None}

\section{REFERENCES}

[1]. Shumway-Cook A, Anson D, Haller S. Postural sway biofeedback: its effect on re-establishing stance stability in hemiplegic patients". Arch. Phys. Med. Rehabil. 1988;69(6):395-400.

[2]. Davidson Madigan, Nussbaum. Effect of lowerExtremity fatigue on Postural Control. European Journal of Applied Physiology. 2004;(92):183-189. 
[3]. Gribble; Hertel. Effect of Lower-Extremity Fatigue on Postural Control. Archives of Physical Medicine and Rehabilitation. 2004;85(4):589-592.

[4]. Kilbreath SL, Raymond J, Refshauge KM. The effect of recurrent ankle inversion sprain and taping on proprioception at the ankle. Med Sci Sports Exerc. 2000;32(1): 10-5.

[5]. Guskiewicz KM, Perrin DH. Effect of orthotics on postural sway following inversion ankle sprain. Journal of Orthopaedic and Sports Physical Therapy. 1996;23(1):326-331.

[6]. Blackburn J Prentice WE Guskiewicz KM Busby MA. Balance and joint stability: the relative contributions of proprioception and muscular strength. J Sport Rehabil. 2009;9(4):315-328.

[7]. O'Sullivan, Susan; Schmitz, Thomas. Physical Rehabilitation (Fifth ed.). Philadelphia: F.A. Davis Company. 2007;pp.254-259.

[8]. Micheal Kent. The Oxford dictionary of Sports Science \& Medicine. 2006. (3r Edition)

[9]. Robert Wood. Y Balance Test. Topend Sport $s$ Website, June 2015, https:// www.topendsports.com.( accessed on 5/1/2021)

[10]. Hrysomallis, C. Balance ability and athletic performance. Sports Medicine. 2011;41 (3): 221-232.

[11]. Eknoyan, Garbed. "Adolphe Quetelet (1796-1874)the average man \& indices of obesity". Nephrology Dialysis Transplantation. 2007;23(1):47-51.

[12]. Swarnalatha S, Sivashankari A, Malarvizhi D. Correlation between body mass index and dynamic postural control among healthy young adults. Biomedical Research \& clinical practice 2018;3(3):1-6.

[13]. Tajwar Yasmeen, Mohammad Anamul Haque, Sanjay Kumar et al. Relationship between Body Mass Index \& Dynamic Balance in elderly population: A correlational study. Journal of emerging technology and emerging research. 2018;5(10):301.
[14]. Jessica Velotta, Josh Weyer \& co Relationship between leg dominance tests \& type of tasks. Portuguese Journal of Sports Science 2011;11(Suppl 2):1037.

[15]. Sadhegi H, Allard P, Prince F, Labelle H. Symmetry $\&$ limb dominance in able-bodied gait: a review. Gait Posture. 2000; Sep;12(1):3-45.

[16]. Nicole Kahle, Phillip A Gribble. Core stability training in dynamic balance testing among young, healthy adults. Athletic training and sports health care $2009 ; 1(2): 65-73$.

[17]. Gracovetsky . S \& Farfan H. Spine (Phila Pa 1976); 1986;Jul-Aug(6): 543-73.

[18]. Kibler W, J, Sciascia. The role of core stability in athletic function. Sports Med. 2006;36-(3):189198.

[19]. Zattara M, Bouisset S. Posturo-kinetic organization during the early phase of voluntary limb movement. J Neurol Neurosurg Psychiatry 1988;51: 956-65.

[20]. Walker C, Brouwer BJ, Culham. EG.Use of Visual feedback in retraining balance following stroke. Phys ther 2000;80:886-895.

[21]. Dault MC, de Haart M, Geurts AC, Arts IM, Nienhuis B. Effects of visual center of pressure feedback on postural control in young \& elderly healthy adults \& stroke patients. Hum Mov Sci 2003;22:221-236.

[22].Timothy J Brindle, J.C. Mizelle, Steven J. Stanhope. Visual and proprioceptive feedback improves joint position senseKnee. Surg Sports Traumatol Arthrosco. 2011;Jan 17(1): 40-47.

How to cite this article:

Tejaswini Padmanabha Suvarna, Joseph Oliver Raj, Nithin Prakash. Correlation between Balance and BMI in Collegiate students: A cross sectional study. Int J Physiother Res 2021;9(1):3759-3764. DOI: 10.16965/ ijpr.2021.103 